\title{
Imaging and tracking HIV viruses in human cervical mucus
}

Fatima Boukari

Sokratis Makrogiannis

Ralph Nossal

Hacène Boukari 


\title{
Imaging and tracking HIV viruses in human cervical mucus
}

\author{
Fatima Boukari, ${ }^{a, b}$ Sokratis Makrogiannis, ${ }^{b, c}$ Ralph Nossal, ${ }^{d}$ and Hacène Boukari ${ }^{b, c, *}$ \\ aDelaware State University, Department of Mathematics, 1200 North Dupont Highway, Dover, Delaware 19901, United States \\ ${ }^{b}$ Delaware State University, Department of Physics and Engineering, 1200 North Dupont Highway, Dover, Delaware 19901, United States \\ 'Delaware State University, Delaware Institute of Science and Technology, 1200 North Dupont Highway, Dover, Delaware 19901, United States \\ 'Eunice Kennedy Shriver National Institute of Child and Human Development, National Institutes of Health, 9000 Rockville Pike, Bethesda, \\ Maryland 20892, United States
}

\begin{abstract}
We describe a systematic approach to image, track, and quantify the movements of HIV viruses embedded in human cervical mucus. The underlying motivation for this study is that, in HIV-infected adults, women account for more than half of all new cases and most of these women acquire the infection through heterosexual contact. The endocervix is believed to be a susceptible site for HIV entry. Cervical mucus, which coats the endocervix, should play a protective role against the viruses. Thus, we developed a methodology to apply time-resolved confocal microscopy to examine the motion of HIV viruses that were added to samples of untreated cervical mucus. From the images, we identified the viruses, tracked them over time, and calculated changes of the statistical mean-squared displacement (MSD) of each virus. Approximately half of tracked viruses appear constrained while the others show mobility with MSDs that are proportional to $\tau^{\alpha}+\nu^{2} \tau^{2}$, over time range $\tau$, depicting a combination of anomalous diffusion $(0<\alpha<0.4)$ and flow-like behavior. The MSD data also reveal plateaus attributable to possible stalling of the viruses. Although a more extensive study is warranted, these results support the assumption of mucus being a barrier against the motion of these viruses. @ 2016 Society of Photo-Optical Instrumentation Engineers (SPIE) [DOI: 10.1117/1.JBO.21.9.096001]
\end{abstract}

Keywords: fluorescence confocal imaging; particle tracking; cervical mucus; HIV virus; constrained random walk. Paper 160086RR received Feb. 11, 2016; accepted for publication Aug. 8, 2016; published online Sep. 6, 2016.

\section{Introduction}

Several reports have shown that the main mode of transmission of AIDS is through exposure of mucosal layers to HIV viruses and HIV-infected cells. ${ }^{1}$ Details on the interactions of the viruses or the cells with the layers, which lead to entry of the viruses across the mucosa, remain unclear. Conceptually, one may posit the role of damaged epithelial barriers as a likely mode of entry for the viruses. However, based on studies of transmission of a similar virus, the simian immunodeficiency virus in nonhuman primates, it appears that HIV viruses are likely to traverse healthy mucosal layers as well. ${ }^{2-8}$ It is then essential to understand how HIV viruses and HIV-infected cells interact with various components of uninfected and undamaged mucosal layers. This is particularly important in developing an understanding of the etiology of HIV infection in women, who constitute more than half of all people living with HIV worldwide ${ }^{9}$ and are most likely to acquire HIV through heterosexual intercourse. Studies of these interactions and the produced results may also be relevant to the efforts for designing vaccines for the prevention of HIV transmission ${ }^{10}$ and new microbicides. ${ }^{11-16}$ The protection of the integrity of these mucosal barriers and their roles may be as important as targeting the HIV viruses or HIV-infected cells. Central to these studies is the development of methodology for tracking and characterizing movements of infectious agents. Tracking techniques typically include a stage of particle localization followed by temporal correspondences of particles. ${ }^{17,18}$ Motion correspondences can be obtained by deterministic approaches using nearest neighbors or graph theory, ${ }^{19}$ or probabilistic approaches such as Kalman and particle filters. ${ }^{20,21}$ In this paper, we demonstrate an optical imaging technique to obtain time-lapse imaging of HIV viruses in mucus. We also demonstrate a deterministic method for subsequent detection and tracking of the viruses and quantification of their motion patterns.

Cervical mucus is a complex biogel made up of water ( $\sim 95 \%)$, gel-forming mucins $(\sim 3 \%)$, and diverse macromolecules such as DNA, lipids, enzymes, and antibodies as well as cells such as squamous cells and bacteria. ${ }^{22-25}$ It is particularly known for its protective role as a biological barrier against pathogens. $^{26,27}$ Recently, we described results of analysis of fluorescence correlation spectroscopy and time-lapse fluorescence confocal microscopy (t-FCM) measurements of the movements of inactivated and fluorescent HIV viruses embedded in vitro in unprocessed, crude cervical mucus provided by healthy donors. ${ }^{1}$ We separately tracked the viruses in each individual mucus sample. Because large variations in the properties of the samples might exist due to, for example, changes over the menstrual cycle, we did not mix the samples. Also, by not mixing, we preserved as much as possible the structural microenvironments that the viruses might encounter while they moved through the samples. We found that the displacements of the viruses within the cervical mucus are much slower (200-fold) than when in aqueous solution. ${ }^{1}$ Time-lapse images indicated that the viruses can be readily trapped within the mucus. Sudden jumps during the motions of the viruses were also observed. 
In this report, we expand our investigation and describe results obtained from the analysis of additional time-resolved fluorescence confocal microscopy measurements, ${ }^{1}$ where we record and track the movements of fluorescently labeled HIV viruses within human cervical mucus samples. In contrast to the time range ( 5 to $10 \mathrm{~s}$ ) used in our previous report, ${ }^{1}$ we extended the recorded time period up to $34 \mathrm{~s}$, thus providing insight into the overall movements of the viruses over a much longer time scale. We tracked 52 viruses that were located in different spots of the mucus sample, and derived changes of the mean-squared displacement (MSD) over the recorded time for each virus. About half of the viruses appeared constrained. In the other half, we noticed multilevel plateaus when MSD was plotted with time. We analyzed the data by simultaneously accounting for two mechanisms, anomalous diffusion and flow-like motion (directed), to assess the mobility (or immobility) of the viruses within the mucus samples. These samples differed from those studied in our earlier work. The emphasis here is on the analysis of virus movement within the gels.

\section{Samples and Methods}

Details of the samples, sample preparation, and methods were described in Ref. 1. Below, we briefly summarize some relevant aspects.

\subsection{Samples}

\subsubsection{Mucus samples}

Specimens of cervical mucus were collected from non-HIV infected, generally healthy, premenopausal female donors by aspiration, using an endometrial suction curette (Unimar Pipelle, Cooper Surgical, Shelton, Connecticut). The samples were carried on ice, stored at $4^{\circ} \mathrm{C}$, and were typically studied within 48 hours from the collection time.

We assessed qualitatively the elasticity of the samples by stretching and also measured the $\mathrm{pH}$ of the samples with $\mathrm{pH}$ paper testing strips. In our study, we only used samples that had been collected in midmenstrual cycle. All showed similar elastic properties, springing back to their original shape, and their $\mathrm{pH}$ was close to neutral, in accord with previously published data. ${ }^{28}$ The mucus specimens were deposited onto a custom-made sample holder (see Sec. 2.1.4) and studied at room temperature (ca. $20^{\circ} \mathrm{C}$ ). Some samples were optically turbid due to the presence of cells (e.g., squamous cells) and other large scatterers, but we were able to find relatively clear spots for imaging. We analyzed four different samples in detail. For each sample, at least five different fields of view were processed on average with each view containing at least 10 viruses. Note that the data presented here in detail are for the sample that was imaged over the longest time $(\sim 30 \mathrm{~s})$.

\subsubsection{Inactivated, fluorescently labeled HIV viruses}

As previously indicated in Ref. 1, HIV-1 viruses (viral strains MN) were inactivated and internally labeled with Alexa Fluor 488 C5 maleimide (Fluorescent Probes, Invitrogen, Eugene, Oregon), to alkylate cysteines of zinc fingers of the HIV-1 nucleocapsid protein. ${ }^{29}$ Since disulfide-bonded cysteines of the envelope glycoproteins were unaffected, the functional properties of the viral envelope were preserved. The purified fluorescent viruses were stored in PBS buffer at $-80^{\circ} \mathrm{C}$. Prior to being studied, the viruses were thawed, gently mixed, and centrifuged at $14,000 \mathrm{rpm}(\sim 16,000 \mathrm{~g})$ in an Eppendorf MicroCentrifuge $5415 \mathrm{C}$ for $3 \mathrm{~min}$ to remove any aggregates formed during storage.

\subsubsection{Polystyrene beads bind to mucus}

By mixing bright fluorescent 28-nm polystyrene beads with a mucus sample, we were able to delineate the overall structure of the mucus. In Fig. 1, we show an image of the beads decorating the mucus sample, which clearly demonstrates the existence of filamentous bundles. This observation calls into question how the viruses, which are much smaller in size $(\sim 100 \mathrm{~nm})$, move in a medium containing these ordered structures.

\subsubsection{Preparation of samples for microscopy}

The sample holders consisted of two glass coverslips (size: A) separated by a $\sim 200-\mu \mathrm{m}$ thick rubber spacer containing a $5-\mathrm{mm}$ diameter hole. To minimize interactions with the viruses, the coverslips were treated with Sigmacote (Sigma-Aldrich, Saint Louis, Missouri), which creates a neutral, hydrophobic thin film on the surface.

A small sample $(\sim 20 \mu \mathrm{l})$ of the mucus was placed in the sample holder and a 1 to $2 \mu$ l aliquot of the concentrated solution of the viruses was added with a micropipette. The viruses were introduced into the mucus by slowly inserting the tip of the pipette into the sample, slowly releasing the virus solution, and gently mixing the two materials.

\subsection{Time-Resolved Fluorescence Confocal Microscopy}

We recorded movements of viruses embedded in the mucus samples using our custom-built confocal microscope system consisting of an IX70 inverted microscope (Olympus, Center

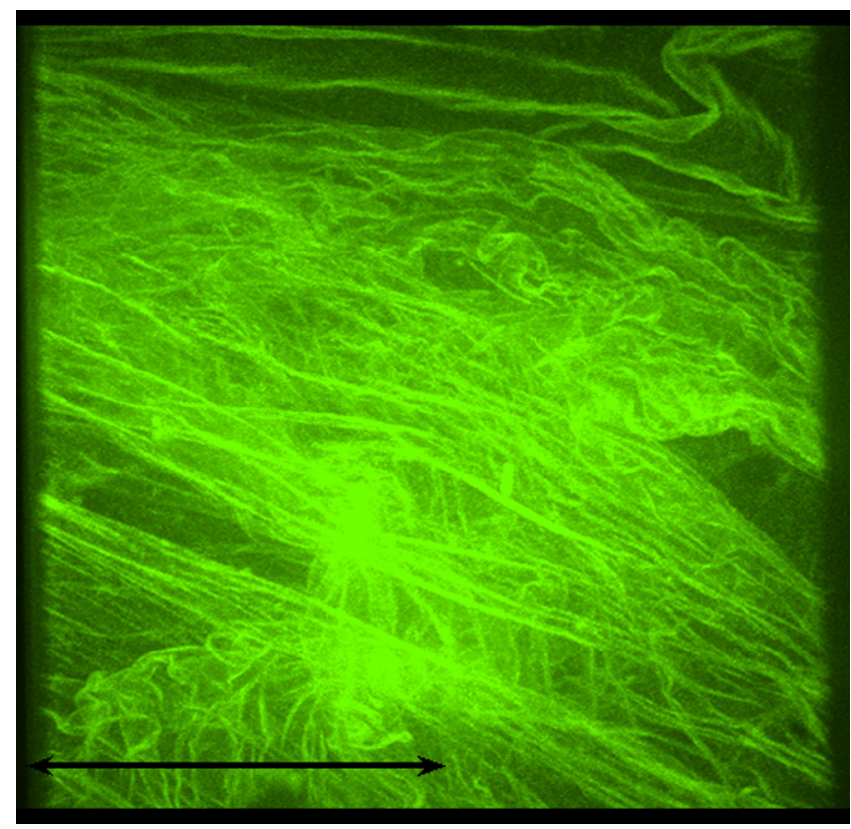

Fig. 1 A $512 \times 512$ digital image of a sample of human cervical mucus labeled with 28 -nm fluorescent polystyrene beads shows filamentous bundles in the mucus. The image, which was taken with a 488-nm laser beam, was generated from stacked confocal images, each being scanned over $100 \mathrm{~ms}$ period. The bar indicates the scale in $40 \mu \mathrm{m}$. 
Valley, Pennsylvania), a confocal spinning disk analyzer (Perkin-Elmer, Fremont, California) attached to a cooled $512 \times$ 512 CCD camera (Model C9100, Hamamatsu, Bridgewater, New Jersey), an air-cooled argon ion laser (Melles-Griot, Carlsbad, California) having two basic excitation lines (488 and $568 \mathrm{~nm}$ ), a piezo-electric device (Physik Instrumente, Karlsruhe/Palmbach, Germany) for adjusting the $z$-position of the objective, and a 2D XY-Proscan stage (Prior, Rockland, Massachusetts). The system was set on a low-vibration isolation table (Newport, Irvine, California), and images were collected with a $60 \times, \mathrm{NA}=1.2$ water Olympus objective. IPLab ${ }^{\mathrm{TM}}$ software (BD Biosciences, Rockville, Maryland) was used to control various devices, and to acquire and analyze data.

We collected 300 sequential frames spanning a period of $34 \mathrm{~s}$. The exposure time for each image was set to $100 \mathrm{~ms}$. For calibration of the pixel size, we used fluorescent spherical beads with known diameters: 5, 10, and $20 \mu \mathrm{m}$. To avoid boundary effects, the observed focal plane was always kept about $50 \mu \mathrm{m}$ away from the surface of the bottom coverslip of the sample holder. We used bright-white transmission illumination to check that the imaged field was free of large features such as cells or unknown debris.

\section{Image Analysis}

The fluorescent viruses appear as bright spots in the digital confocal images with a matrix size of $512 \times 512$ pixels. In the field of view, we typically observed 10 to 15 viruses. In Fig. 2, we show an image of bright spots attributed to the fluorescent viruses, generated by collapsing a collection of z-stacked confocal images. This procedure increased the contrast between the viruses and the mucus background, revealing all the viruses that are in a thick layer of sample $(\sim 100 \mu \mathrm{m})$ within the field of view.

Using a custom algorithm encoded in MATLAB programming language, we tracked each virus and determined changes of the spatial pixel-coordinates $[X(t), Y(t)]$ of the center of mass (centroid) of its intensity profile with time. To track the viruses, we first used nonlinear diffusion filtering to reduce the

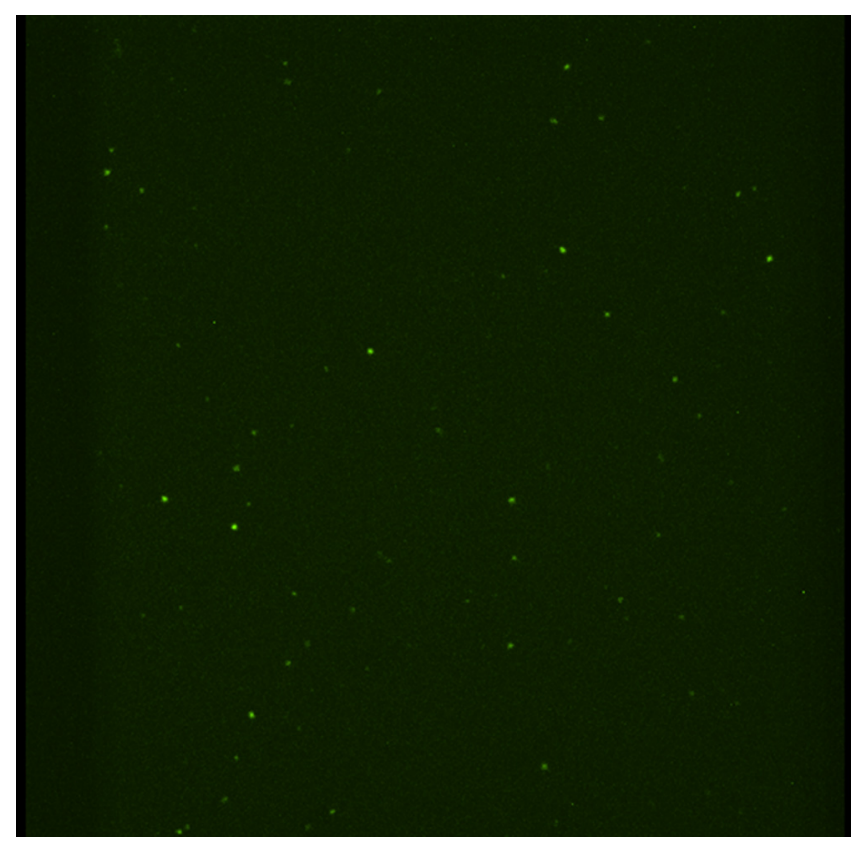

Fig. 2 Fluorescent viruses dispersed in a sample of cervical mucus. underlying noise and then computed the photocount histogram of the image. Next, we determined a user-interactive intensity threshold for the whole sequence to detect virus candidate regions. After thresholding, the viruses were identified as components of a size larger than 8 pixels in order to exclude false positives that may be caused by remaining noise. At each time frame, we computed the centroids of the viruses in each image that we used for representation and tracking of each virus component. We initialized each track by the spatial location of the centroid of each virus in the first frame. Then, a frame-by-frame association of nearest neighbors was employed to track the viruses. The $(X, Y)$ coordinates of the center of mass and the highest and average intensities of each virus were determined and automatically stored. Typically, 10 to 15 viruses were detected in a single field of view and were tracked along with their maximum and spatially averaged intensities.

Figure 3 shows the analysis of motion of a virus embedded in cervical mucus over a 34-s time interval. More specifically, Fig. 3(a) displays the $(X, Y)$ tracks of the virus in pixel fraction. At first glance, the motion pattern indicates likely trapping within the host medium over the 300 time frames in the data set. For comparison, the viruses exhibit a much higher degree of mobility when in buffered solutions-not shown hereand often move away from the confocal field of view. Along with the $(X, Y)$ tracks, we assess the stability of the intensity profile over a consecutive sequence of frames (300 frames over $34 \mathrm{~s}$ ). Systematic or sudden changes of the intensity are a measure of photobleaching or movement of the virus away from the initial $(X, Y)$ confocal plane along the vertical $z$-orientation. In Fig. 3(b), we plot the maximum fluorescent intensity, as a function of frame number (time), of the virus whose movement is shown in Fig. 3(a). Figure 3(c) shows the histogram of the maximum intensity over the 300 frames of this virus. Together, Figs. 3(b) and 3(c) show minimal spatial drift away from the original confocal plane. If the virus had moved out of the image plane, the distribution in Fig. 3(c) would have had greater asymmetry.

We followed the approach detailed in Ref. 30 to assess the movement of each individual virus by first calculating the MSD of the virus particle expressed by the following equation:

$$
\begin{aligned}
\operatorname{MSD}(\tau) & =\langle X(t+\tau)-X(t)\rangle^{2}+\langle Y(t+\tau)-Y(t)\rangle^{2} \\
& \equiv\left\langle\Delta X^{2}+\Delta Y^{2}\right\rangle
\end{aligned}
$$

where $t, \tau,\langle\rangle$ denote the time, the "delay time," and the time average as described in Ref. 1, respectively. Typically, the MSD in Eq. (1) can be considered the amount of space each virus has "explored" in the host mucus over the observation time. In Fig. 3(d), we plot the MSD of the virus whose tracks are shown in Fig. 3(a), as well as the $\Delta X^{2}$ and $\Delta Y^{2}$ components of this quantity. Now, we notice a systematic slow motion of the virus and then an apparent stalling of movement in the $X$-direction between 12 and $18 \mathrm{~s}$ before movement in that direction resumes. The initial part of the MSD $(<15 \mathrm{~s})$ of the virus in Fig. 3(d) shows a positive curvature. We surmise that the movement of this type of virus is governed by two mechanisms: anomalous diffusion with MSD proportional to a power law $\left(\tau^{\alpha}\right)$ and flow-like behavior with MSD proportional to $\tau^{2}$. Anomalous diffusion has received attention within the literature associated with single particle tracking. ${ }^{31}$ In particular, it is used to describe transport scenarios within crowded systems such as actin filament network ${ }^{32}$ and cytoplasms $s^{33,34}$ and it has been 
(a)

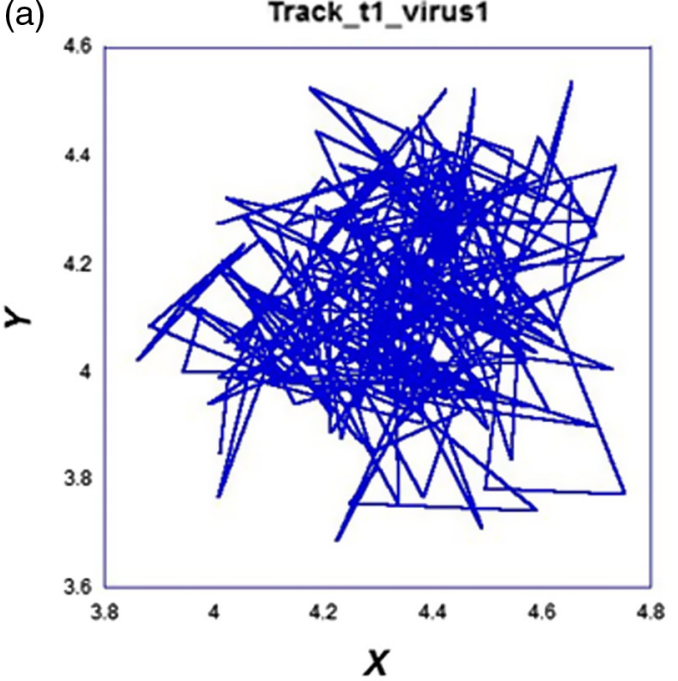

(c)

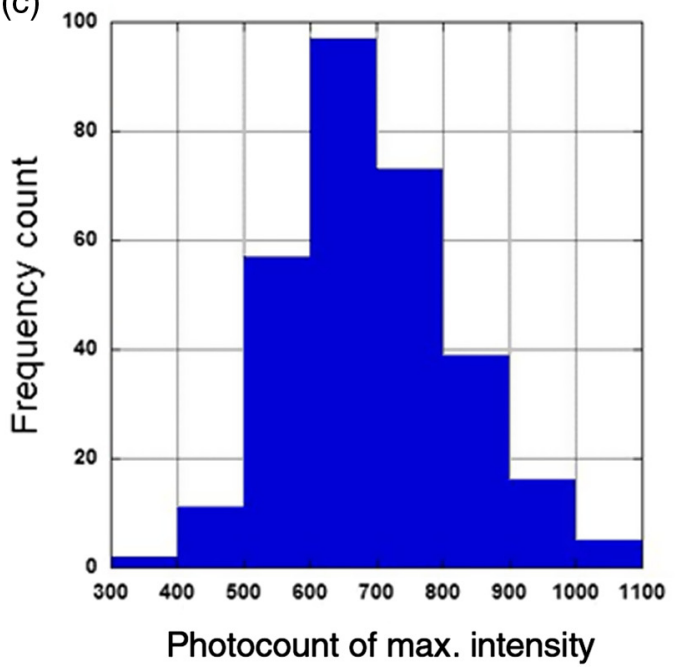

(b)

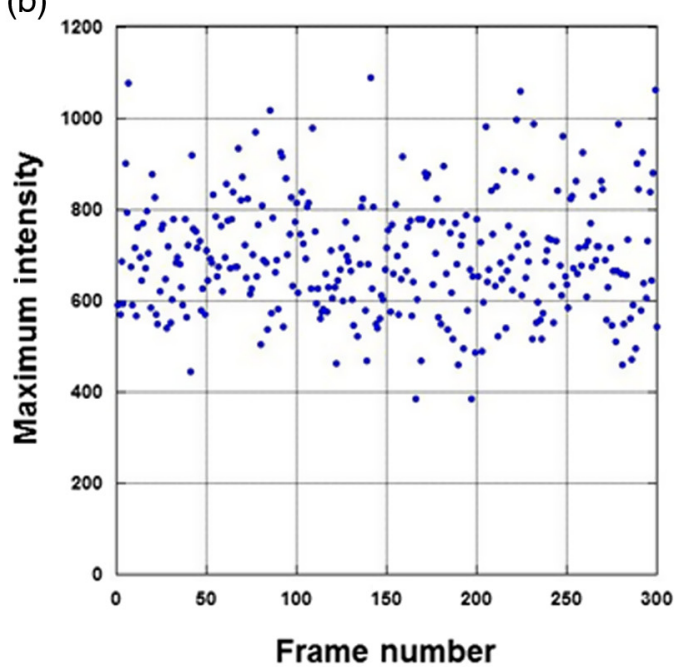

(d)

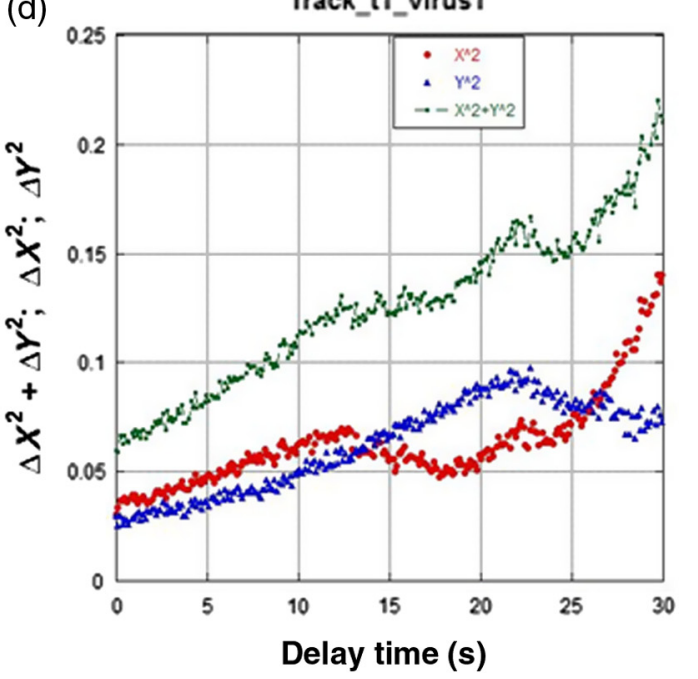

Fig. 3 (a) $(X, Y)$ track of a virus embedded in a sample of cervical mucus over a 34 s recording time (300 frames); (b) fluctuations of the maximum intensity against the frame number of the peak associated with the virus (a), indicating stability of the intensity over the collection time; (c) photocount histogram corresponding to (b) over the 300 frames. The approximate symmetry of the photocount histogram is indicative of stability of the intensity over the recorded period, signifying that the virus stayed essentially in a single $X-Y$ confocal field of view; (d) the MSD $\left\langle\Delta X^{2}+\Delta Y^{2}\right\rangle$ of the virus shown in (a), plotted as a function of delay time (up to $30 \mathrm{~s}$ ). The components $\Delta X^{2}$ and $\Delta Y^{2}$ of the overall displacement are also plotted.

proposed as a measure of macromolecular crowding. ${ }^{33}$ We also include directed motion (flow-like) likely due to structural reorganization of mucus (e.g., shrinking or stretching) over time. Here, we assume that this reorganization can be described by a linear behavior within a short period of time $(<15 \mathrm{~s})$.

If the two mechanisms are not coupled, the MSD of the virus can be described by the following expression:

$$
\operatorname{MSD}=\Gamma \tau^{\alpha}+\nu^{2} \tau^{2},
$$

where $\alpha$ describes anomalous diffusion for $\alpha<1$, the factor $\Gamma$ is a constant, and $v$ characterizes the apparent velocity of the directed motion (flow-like). For $\alpha=1$, the expression in Eq. (2) describes an uncoupled, normal diffusion-flow dynamics. ${ }^{25}$ Figure 3 provides additional insight beyond what was discussed in our previous report. ${ }^{1}$ In particular, there is the possibility for the virus to be caged temporally for discrete periods of time, as indicated by the first plateau (12 to $18 \mathrm{~s}$ ) and the second plateau (22 to $26 \mathrm{~s}$ ), displayed in Fig. 3(d). The virus appears to regain its mobility between the two plateaus and after, when it escapes from both "cages."

However, the behavior of the virus depicted by Figs. 3(a)-3(d) is not universal. For comparison, we show in Figs. 4(a) and 4(b) the behavior of another virus particle, observed in the same field of view. While the $(X, Y)$ tracks in Fig. 4(a) have patterns that are similar to those shown in Fig. 3(a), the time-dependent MSD that we display in Fig. 4(b) is significantly different. Here, the MSD indicates no systematic overall movement of the virus. Rather, it appears constrained with $\left\langle\Delta X^{2}+\Delta Y^{2}\right\rangle,\left\langle\Delta X^{2}\right\rangle$, and $\left\langle\Delta Y^{2}\right\rangle$ being constant, independent of the lag time.

Based on the time-dependence of the MSD, we classified the behavioral dynamics of all viruses observed and tracked in four different fields of view. We found that about $52 \%$ of the viruses appear to be moving, albeit very slowly. The rest $(\sim 48 \%)$ appear 
(a)

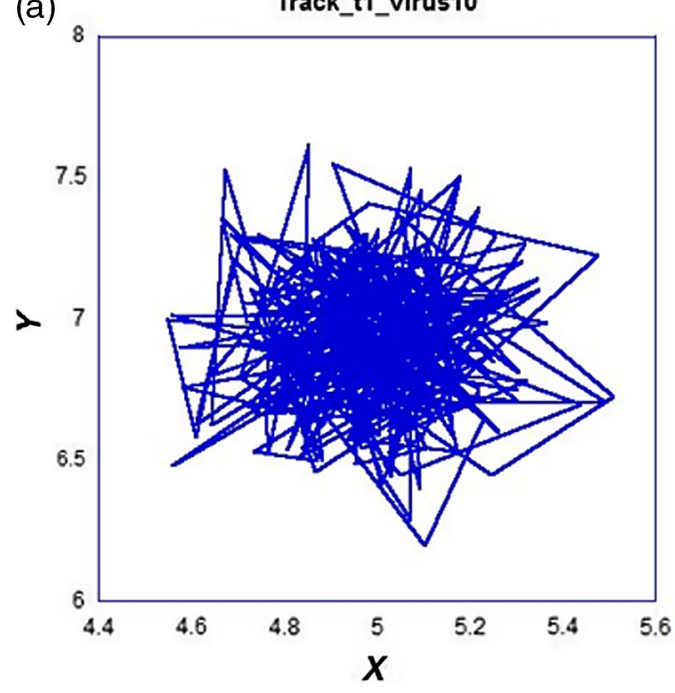

(b)

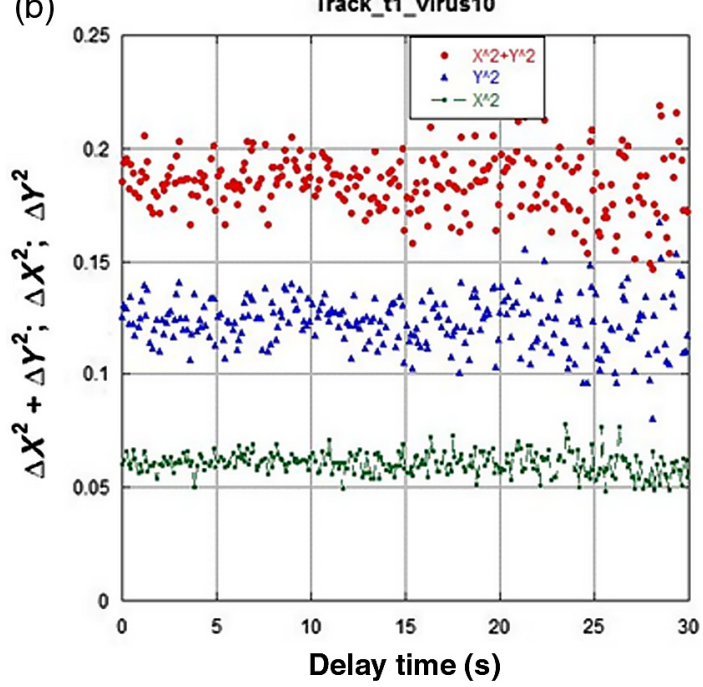

Fig. 4 (a) $(X, Y)$ tracks of a different virus observed in the same field of view as the peak in Fig. 3(a), also indicating trapping; (b) MSD $\left\langle\Delta X^{2}+\Delta Y^{2}\right\rangle$ of the virus, plotted as a function of delay time. Together with the $\Delta X^{2}$ and $\Delta Y^{2}$ components of the overall displacement, one notices no net movement over time. Comparison of the overall MSD of this virus with that shown in Fig. 3(d), probably indicating differences in the structure or charge of the virus, as well as local mucus microenvironments.

to be constrained, with their MSD being independent of, or very weakly dependent on, time over a time period of $25 \mathrm{~s}$.

\section{Discussion and Conclusions}

We have focused on the interactions of HIV viruses with human cervical mucus, and have studied the possible movement of the viruses within the mucus. The underlying motivation is that women account for more than half of all new cases in HIVinfected adults, and most of these women acquire the infection through heterosexual contact. ${ }^{2}$ It is well established that both free and cell-associated HIV viruses can infect the vaginal, ectocervical, and endocervical mucosa. The endocervix has been pointed out as a particularly susceptible site for HIV-virus entry because it is lined with a single layer of columnar epithelium. Further, the region where the ectocervix transforms into the endocervix can be populated with CD4+ T-cells. ${ }^{35}$ Several investigators have speculated on the possible pathways exploited by the HIV viruses for entry once they reach the epithelial layer. ${ }^{2,35,36}$ However, the viruses would have to traverse the cervical mucus layer ( $\sim 0.5$ to $1 \mathrm{~mm})$ before making contact with the epithelial layer.

We have described a methodology that provides insight into the structure of cervical mucus and the movements of viruses embedded in samples of mucus. Here, we exploited, in particular, the fluorescence property of fluorescently labeled polystyrene nanobeads to reveal the filamentous structure of the mucus sample (see Fig. 1). We also used the fluorescence of the fluorescently labeled and inactivated HIV viruses that were mixed in samples of mucus (Fig. 2) to detect the viruses and track their movements. The host mucus was not fluorescent and hence "invisible," and the bright fluorescent spots in Fig. 2 are related to the viruses.

Our study confirmed that cervical mucus is very heterogeneous, as others have observed. ${ }^{37-40}$ As reported in Ref. 1, the mucus slowed the movement of the viruses, about 200fold as compared to that of the viruses in aqueous solution. Compared to the previous report, we doubled the observation time $(\sim 30 \mathrm{~s})$ and found that the mucus appears to trap the viruses in a way similar to that reported in Ref. 26. However, they were not completely immobile. Comparison of the MSDs of the viruses shows significant differences in the mobility or immobility of each individual virus. The MSDs of the individual viruses showed a combination of two mechanisms of motion, anomalous diffusion and directed-like motion (flow-like), as well as possible stalling over time.

In our analysis, we avoided the use of ensemble averaging of all MSDs, in contrast to what is commonly done in statistical analysis of movements of particles derived from imaging techniques. Averaging tends to blunt differences in viral motion, which are attributable to the spatial heterogeneity of mucus (see Fig. 1) and/or the structural and surface characteristics of the viruses. Some viruses show anomalous diffusion over a wide range of time, with their MSDs proportional to $\tau^{\alpha}$ with $\alpha \in[0,0.4]$, whereas others show a combination of anomalous diffusion and directed-like movement $\left(\mathrm{MSD}=\Gamma \tau^{\alpha}+\nu^{2} \tau^{2}\right)$. Several physical and chemical processes can be suggested to account for these mechanisms, including thermal fluctuations and structural rearrangement of the mucus due to stretching or other mechanical perturbations. The underlying mechanisms for the observed anomalous subdiffusion $(\alpha<1)$ remain the subject of ongoing investigations. For example, Wong et al. ${ }^{32}$ reported measurements of polystyrene beads diffusing in entangled F-actin networks and observed anomalous subdiffusion of their probes when the bead size was close to the lattice mesh size. They argued that this anomalous diffusion results from discrete, rapid movement from one microscopic site to another; without these jumps, the probes would have been constrained, resulting in an MSD independent of time. Similarly, the combination of constrained and moving viruses in this study may explain the subdiffusion process.

Analysis of time-lapse images of viruses embedded in cervical mucus demonstrates significant interactions between the mucus and the viruses, confirming the barrier role of mucus. Mucus, together with innate antiviral proteins, may be a 
"gatekeeper" that, under appropriate physiological conditions, selectively inhibits the transmission of HIV-1 viruses. ${ }^{41}$ Overall, the viruses do not readily move within the mucus samples. Thus, it may be that mechanical perturbations, present during coitus, are a major mechanism leading to infection. A comprehensive study that examines the mechanical properties of mucus, especially in light of changes that occur in cervical mucus and other vaginal secretions during the menstrual cycle, might be informative. The effects of temperature, $\mathrm{pH}$, salt concentrations, and other environmental factors should also be systematically studied, perhaps to understand how simple prophylactic additives might stiffen the gel and/or change gel-virus interactions and thereby affect the likelihood of transmitting the disease. Maintaining the integrity of the protective role of mucus is of clinical relevance, especially in the design of antiviral drugs.

\section{Acknowledgments}

F. Boukari, S. Makrogiannis, and H. Boukari received support from the Center for Research and Education in Optical Sciences and Applications (CREOSA) at Delaware State University funded by NSF (HRD-1242067), from NASA Grant \#NNX09AU90A, and from NIH Delaware INBRE (P20 RR016472). Research reported in this publication was also supported by the National Institute of General Medical Sciences under Award Number SC3GM113754. R. Nossal was supported by intramural funds from the Eunice Kennedy Shriver National Institute of Child Health and Human Development. We thank our NIH colleagues, Dr. Beda Brichacek, Dr. Jeffrey Lifson, Dr. Leonid Margolis, Dr. Sheila Mahoney, and Dr. Pamela Stratton for their earlier participation in this project.

\section{References}

1. H. Boukari et al., "Movements of HIV-virions in human cervical mucus," Biomacromolecules 10, 2482-2488 (2009).

2. P. A. Kozlowski and M. R. Neutra, "The role of mucosal immunity in prevention of HIV transmission," Curr. Mol. Med. 3, 217-228 (2003).

3. C. D. Pauza et al., "Pathogenesis of SIVmac251 after atraumatic inoculation of the rectal mucosa in rhesus monkeys," J. Med. Primatol. 22, 154-161 (1993).

4. A. I. Spira et al., "Cellular targets of infection and route of viral dissemination after an intravaginal inoculation of simian immunodeficiency virus into rhesus macaques," J. Exp. Med. 183, 215-225 (1996).

5. Z.-Q. Zhang et al., "Sexual transmission and propagation of SIV and HIV in resting and activated CD4+ T cells," Science 286, 1353-1357 (1999).

6. C. J. Miller et al., "Pathology and localization of simian immunodeficiency virus in the reproductive tract of chronically infected male rhesus macaques," Lab. Invest. 70, 255-262 (1994).

7. C. Stahl-Hennig et al., "Rapid infection of oral mucosal-associated lymphoid tissue with simian immunodeficiency virus," Science $\mathbf{2 8 5}$, 1261-1265 (1999).

8. T. W. Baba et al., "Mucosal infection of neonatal rhesus monkeys with cell-free SIV," AIDS Res. Hum. Retroviruses 10, 351-357 (1994).

9. The Foundation for AIDS Research, http://www.amfar.org/ (25 August 2016).

10. International AIDS Vaccine Initiative, http://www.iavi.org/ (25 August 2016).

11. S. Rerks-Ngarm et al., "Vaccination with ALVAC and AIDSVAX to prevent HIV-1 infection in Thailand," N. Engl. J. Med. 361, 22092220 (2009).

12. M. L. Robb et al., "Risk behaviour and time as covariates for efficacy of the HIV vaccine regimen ALVAC-HIV (vCP1521) and AIDSVAX B/E: a post-hoc analysis of the Thai phase 3 efficacy trial RV 144," Lancet Infect. Dis. 12, 531-537 (2012).
13. J. Jardine et al., "Rational HIV immunogen design to target specific germline B cell receptors," Science 340, 711-716 (2013).

14. A. B. Balazs et al., "Vectored immunoprophylaxis protects humanized mice from mucosal HIV transmission," Nat. Med. 20, 296-300 (2014).

15. M. R. Gardner et al., "AAV-expressed eCD4-Ig provides durable protection from multiple SHIV challenges," Nature 519, 87-91 (2015).

16. D. H. Barouch et al., "Protective efficacy of a global HIV-1 mosaic vaccine against heterologous SHIV challenges in rhesus monkeys," Cell 155, 531-539 (2013).

17. W. J. Godinez et al., "Deterministic and probabilistic approaches for tracking virus particles in time-lapse fluorescence microscopy image sequences," Med. Image Anal. 13(2), 325-342 (2009).

18. E. Meijering, O. Dzyubachyk, and I. Smal, "Methods for cell and particle tracking," Methods Enzymol. 504, 183-200 (2012).

19. I. Sbalzarini and P. Koumoutsakos, "Feature point tracking and trajectory analysis for video imaging in cell biology," J. Struct. Biol. 151(2), 182-195 (2005).

20. Z. Khan, T. Balch, and F. Dellaert, "MCMC-based particle filtering for tracking a variable number of interacting targets," IEEE Trans. Pattern Anal. Mach. Intell. 27(11), 1805-1819 (2005).

21. I. Smal et al., "Particle filtering for multiple object tracking in dynamic fluorescence microscopy images: application to microtubule growth analysis," IEEE Trans. Med. Imaging 27(6), 789-804 (2008).

22. I. Carlstedt et al., "Quantitation of mucus glycoproteins blotted onto nitrocellulose membranes," Biochem. J. 211, 13-22 (1983).

23. J. K. Sheehan and I. Carlstedt, "Size heterogeneity of human cerical mucus glycoproteins. Studies performed with rate-zonal centrifugation and laser light scattering," Biochem. J. 245(3), 757-762 (1987).

24. R. Bansil and B. S. Turner, "Mucin structure, aggregation, physiological functions and biomedical applications," Curr. Opin. Colloid Interface Sci. 11(2-3), 164-170 (2006).

25. J. L. V. Shaw, C. R. Smith, and E. P. Diamandis, "Proteomic analysis of human cervico-vaginal fluid," J. Proteome Res. 6(7), 2859-2865 (2007).

26. D. Maher et al., "HIV binding, penetration, and primary infection in human cervicovaginal tissue," Proc. Natl. Acad. Sci. U. S. A. 102, 11504-11509 (2005).

27. A. M. Fox-Canale et al., "Human cytomegalovirus and human immunodeficiency virus type-1 co-infection in human cervical tissue," Virology 369, 55-68 (2007).

28. C. H. M. Correa, A. L. G. Mattos, and A. N. Ferrari, "In situ variation of cervical mucus pH during exposure to atmospheric air," Braz. J. Med. Biol. Res. 34, 767-770 (2001).

29. D. R. Morcock et al., "Elimination of retroviral infectivity by N-ethylmaleimide with preservation of functional envelope glycoproteins," $J$. Virol. 79, 1533-1542 (2005).

30. H. Qian, M. P. Sheetz, and E. L. Elson, "Single particle tracking: analysis of diffusion and flow in two-dimensional systems," Biophys. J. 60, 910-921 (1991).

31. R. Metzler et al., "Anomalous diffusion models and their properties: non-stationarity, non-ergodicity, and ageing at the centenary of single particle tracking," Phys. Chem. Chem. Phys. 44, 24128-24164 (2014).

32. I. Y. Wong et al., "Anomalous diffusion probes microstructure dynamics of entangled F-actin networks," Phys. Rev. Lett. 92, 178101 (2004).

33. M. Weiss et al., "Anomalous subdiffusion is a measure for cytoplasmic crowding in living cells," Biophys. J. 87, 3518-3524 (2004).

34. I. Golding and E. C. Cox, "Physical nature of bacterial cytoplasm," Phys. Rev. Lett. 96, 098102 (2006).

35. F. Hladik and M. J. McElrath, "Setting the stage: host invasion by HIV," Nat. Rev. Immunol. 8(6), 447-457 (2008).

36. S. R. Galvin and M. S. Cohen, "The role of sexually transmitted diseases in HIV transmission," Nat. Rev. Microbiol. 2, 33-42 (2004).

37. S. S. Olmstead et al., "Diffusion of macromolecules and virus-like particles in human cervical mucus," Biophys. J. 81, 1930-1937 (2001).

38. W. M. Saltzman et al., "Antibody diffusion in human cervical mucus," Biophys. J. 66, 508-515 (1994). 
39. H. Shen, Y. Hu, and W. M. Saltzman, "DNA diffusion in mucus: effect of size, topology of DNAs, and transfection reagents," Biophys. J. 91, 639-644 (2006).

40. S. K. Lai et al., "Rapid transport of large polymeric nanoparticles in fresh undiluted human mucus," Proc. Natl. Acad. Sci. U. S. A. 104, 1482-1487 (2007).

41. L. Margolis and R. Shattock, "Selective transmission of CCR5-utilizing HIV-1: the 'gatekeeper' problem resolved?" Nat. Rev. Microbiol. 4, 312-317 (2006).

Fatima Boukari is a graduate student in the Department of Mathematics at Delaware State University. Her current research interests include the development and application of mathematical tools and computational techniques for image analysis, especially biomedical images.
Sokratis Makrogiannis is an assistant professor in the Department of Physics and Engineering at Delaware State University. His research interests include image analysis and machine learning techniques with applications to biomedicine.

Ralph Nossal recently retired as a senior investigator at the National Institutes of Health but maintains ties to that institution. Among his various research interests are applications of biophotonics to assess the physiological properties of tissues and the physical attributes of tissue components.

Hacène Boukari is an associate professor of physics at Delaware State University and works currently on the development and application of optical and imaging techniques as well as physics-based modeling for understanding the behavior of biologically relevant nanoparticles in crowded and complex environments. 